\title{
ANTECEDENTES Y MOTIVOS DEL PLAN MAESTRO DE DESARROLLO ESPIRITUAL
}

Mg. IsRael Joselito Fernández Mallma Morales, San Martín - Perú 


\title{
Resumen
}

Antecedentes y motivos del Plan Maestro de Desarrollo Espiritual. El desarrollo espiritual ha sido siempre un eje central en la educación adventista. Los propósitos educacionales han sido claramente delineados desde sus inicios por los escritos de Elena G. de White y la teología adventista. El crecimiento de las instituciones tanto numérico como organizativamente demandan una apropiada evaluación de los esfuerzos educacionales enfocados el desarrollo espiritual de sus estudiantes. El Plan Maestro de Desarrollo Espiritual (PMDE) debería reflejar estos propósitos. El proceso de búsqueda por hacer de la espiritualidad un factor relevante o el más trascendente en la educación adventista, y sin importar el nivel de educación al que se aplique, sigue siendo un desafío para los administradores, directivos, capellanes y profesores. Los principios filosófico-bíblicos del crecimiento espiritual requieren ser traducidos a la experiencia práctica de modo que la planificación permita establecer indicadores claros que puedan ser medidos y evaluados.

Palabras clave: PMDE, espiritualidad, evaluación, educación adventista.

\begin{abstract}
Background and Motifs for the Spiritual Master Plan. The spiritual development has always been a pivotal axis for Adventist education. The educational purposes have been clearly established from the very beginning by Ellen G. White writings and the Adventist theology. The numerical and organizational growth of the institutions require an appropriate assessment of the educational efforts focused on spiritual growth of the students. The Spiritual Master Plan (SMP) should reflect those purposes. The search process for making the spirituality a relevant factor or the most transcendent one in Adventist education, and despite the educational level applied to, is still a challenge for administrators, leaders, chaplains, and teachers. The philosophic-biblical principles for spiritual growth require being transferred to a practical experience so that the planning process would allow the establishment of clear indicators for their measurement and evaluation.
\end{abstract}

Keywords: SMP, spirituality, assessment, Adventist education.

Recibido: 05/03/2020

Aceptado: 03/12/2020 


\title{
ANTECEDENTES Y MOTIVOS DEL Plan MaEsTro de Desarrollo ESPIRITUAL
}

\author{
Mg. ISRAEL JoseltTo FERNÁNDEZ MALLMA \\ Morales, San Martín - Perú
}

\section{El desarrollo espiritual}

El desarrollo espiritual es un tema de interés para la educación adventista. Debido a que su misión es "restaurar en el hombre la imagen de su Hacedor $^{\prime \prime} 1$, se realizan diversos esfuerzos para conseguir este propósito. El desarrollo espiritual es un tema que podemos rastrear a través de las Sagradas Escrituras, las que nos presentan un paradigma claro respecto a las personas que entran en contacto con la divinidad. Así, entendemos por desarrollo espiritual el crecimiento de la fe en Dios que experimenta un individuo y que modifica su manera de pensar y actuar (Ro 12:1; Ef 4:22-30; 2 Co 5:17).

Las referencias al corazón y la mente son abundantes en la Biblia, lo que revela que hay un conflicto que ocurre en la cosmovisión del individuo que está en desarrollo espiritual (1 Co 9:25-27; 2 Ti 4:7-8; Ro 8:37). En consecuencia, se espera una transformación en su conducta que integra cada aspecto de su vida personal y que se expresa en actos litúrgicos de carácter religioso y en su relación con sus semejantes y su entorno en general. Este proceso de desarrollo espiritual puede comenzar desde la infancia, cómo está estipulado en la ley de Moisés: "y las repetirás a tus hijos" (Dt 6:7-9) y que es refrendado por el proverbio "instruye al niño en su camino" (Pr 22:6), así como en las cartas del apóstol Pablo: "criadlos en la disciplina e instrucción del Señor" (Ef 6:4).

Sin embargo, aunque criados en la fe de sus padres, los jóvenes necesitan ser acompañados y guiados para mantenerse en la fe y no apartarse del camino en el que fueron enseñados, como le pasó en repetidas ocasiones al

Elena G. de White, La educación (Doral, FL: Asociación Publicadora Interamericana, 2009), 15. 
pueblo de Israel: "Y se levantó después de ellos otra generación que no conocía a Jehová, ni la obra que él había hecho por Israel" (Jue 2:10). De modo que la fe en la vida práctica demanda necesariamente un vínculo de relación, como reza el salmo "mi alma tiene sed de ti" (Sal 42:2), y al que el ser humano accede mediante actos litúrgicos provistos por una religión que ha sido construida a partir de la revelación divina de carácter sobrenatural (Éx 31:18). Más aún, se debe considerar que toda relación puede crecer y profundizarse o, de lo contrario, perderse el vínculo entre las partes: “Oíd, cielos, y escucha, tierra, porque el SEÑOR habla: Hijos crié y los hice crecer, mas ellos se han rebelado contra mí" (Is 1:2).

\section{Interés por el desarrollo espiritual en casas superiores de estudio}

Bajo la cosmovisión bíblica, mostrada en las líneas anteriores, se asume que existe la necesidad de cuidar a las nuevas generaciones de creyentes jóvenes para que sea posible su desarrollo espiritual de relación con Dios. En sintonía con esta reflexión, no podemos negar que en la actualidad existen instituciones de educación superior que son patrocinadas por organizaciones de diferentes iglesias cristianas establecidas en diversas partes del mundo. Éstas responden a la misión o intereses de sus promotoras.

Por ejemplo, la Iglesia Católica señala en su Constitución Apostólica sobre Universidades Católicas, que éstas responden al objetivo de "garantizar de forma institucional una presencia cristiana en el mundo universitario" 2 con la tarea de "investigar con libertad, buscar y presentar la verdad, además de promover a través de su influencia los valores y fe que profesan, y que se apoyan en la integración de la fe y la cultura, la identidad católica de la institución y el testimonio personal de sus empleados". ${ }^{3}$ Aunque existen por lo menos tres tipos de universidades católicas, éstas deben responder con lealtad a los propósitos de su promotora. ${ }^{4}$

Por otro lado, tenemos el pronunciamiento de la Asociación de Docentes de Colegios Superiores Luteranos sobre las instituciones estadounidenses de esta denominación. Se ha mostrado la preocupación acerca de dichas instituciones superiores pues estaban operando sin una clara filosofía de

2 Fernando Pérez-Llantada, “Constitución apostólica sobre universidades católicas: Su Santidad Juan Pablo II", Revista de la Facultad de Derecho 42 (1991): 46-48.

3 Ibíd., 48.

4 Juan Manuel Mora, “Universidades de inspiración cristiana: Identidad, cultura, comunicación", Romana 54 (2012): 194-220. 
educación luterana o cristiana distintiva, y que simplemente habían imitado los patrones seculares a los cuales habían añadido cultos, clases de religión y una atmosfera religiosa. ${ }^{5}$ Esto evidencia la existencia del interés en los resultados espirituales que su programa de educación tiene sobre sus estudiantes.

Entre los desafíos que las universidades denominacionales han tenido que enfrentar para sostener sus propósitos filosóficos fundacionales ha sido el lento y sigiloso proceso de secularización. Este proceso no sólo ha socavado la filosofía educacional de instituciones religiosas sino que además las sumergió en un proceso agonizante de aculturación. Appleyard observa que:

es bien sabido que la mayoría de colegios y universidades fundados en los Estados Unidos antes del siglo XX tenían un carácter fuertemente religioso, generalmente protestante cristiano, y que virtualmente todas estas instituciones no tienen una identidad religiosa significativa hoy en día. El ejemplo más conocido es Harvard, fundado por la aprobación de un "ministerio erudito" cuyo lema durante tres siglos fue "Christo et Ecclesiae" (Iglesia de Cristo), pero decenas de otras instituciones incluyendo Yale, Princeton, Chicago, Stanford, Duke, Boston University, e incluso las universidades estatales financiadas con fondos públicos como Michigan y California, tenían un carácter cristiano pronunciado en los primeros años de su existencia y lo abandonaron en el siglo XX. ${ }^{6}$

Evidentemente había un interés religioso en estas instituciones y por tanto se esperaba resultados en ese mismo sentido. La pérdida de su identidad religiosa (que estaba ligada a una denominación) habría sido el resultado del proceso de secularización, que incluía entre otros aspectos, el abandono de pruebas religiosas para la contratación de profesores, la separación legal entre la institución y la organización religiosa, la liberalidad de la teología

5 Esta preocupación parece indicar una especia de máscara o careta cristiana en los sistemas de educación superior en al menos dicha denominación. George R. Knight, “La filosofía de la educación adventista", Revista Educación Adventista 33 (2012): 25.

6 J. A. Appleyard, recensión de The Soul of the American University: From Protestant Establishment to Established Nonbelief, por George M. Marsden, International Higher Education 10, no. 4 (1996): 20. 
convirtiéndose en ciencia moral. ${ }^{7}$ El punto es que existe la necesidad de conocer si una institución está cumpliendo los objetivos espirituales por las cuales fue fundada.

\section{La preocupación adventista por el desarrollo espiritual en sus instituciones}

Las universidades adventistas, alrededor del mundo, han sido fundadas bajo la filosofía de la educación cristiana apoyada firmemente en las Escrituras y en los consejos de Ellen G. White, quien es considerada entre los adventistas del séptimo día como profeta y cofundadora de la Iglesia Adventista del Séptimo Día (IASD). Entre sus más citadas declaraciones sobre la educación, ella escribió que la educación no se limita solo a un determinado curso de estudios, sino que es el "desarrollo armonioso de las facultades físicas, mentales y espirituales". ${ }^{8}$ En sintonía con otros pilares de la educación adventista, las instituciones de nivel superior además de escuelas y colegios alrededor del mundo buscan mantener a flote los principios que le dieron origen. ${ }^{9}$

De acuerdo con la filosofía de la educación adventista, el objetivo de las instituciones del nivel superior además de educar y formar profesionales altamente competitivos, es restaurar en el ser humano la imagen de Dios, y prepararlos para el encuentro con su Salvador. ${ }^{10}$ Para hacer esto, se requiere de manera imperiosa, contar con un programa de desarrollo espiritual. De lo contrario, el desarrollo espiritual solo será una utopía y está condenado al fracaso espiritual.

Por algún tiempo se adoleció de un programa específico al respecto, lo que despertó preocupación entre los líderes. George R. Knight observó este punto y escribió al respecto: "Si las instituciones adventistas del séptimo día son cristianas sólo en el sentido de que tienen a Jesús y el evangelio

7 Ibíd, 20-21. Para mayores detalles, véase Bruce Kuklick, reseña de The Soul of the American University: From Protestant Establishment to Established Nonbelief, por George M. Marsden, Method \& Theory in the Study of Religion 8, no. 1 (1996): 79-84.

8 White, La educación, 14.

9 Office of Archives, Statistics, and Research, Yearbook 2015 (Nampa, ID: Pacific Press Publishing Association, 2015), 5.

10 Knight, “La filosofía de la educación adventista”, 27. 
protestante, entonces vale cualquier escuela evangélica". ${ }^{11}$ Estas palabras ilustran de algún modo que existe la necesidad de fortalecer los programas espirituales alineados a la filosofía de la educación adventista que permitan alcanzar sus altos objetivos. Es así que para 1999, ya comenzaban a surgir escritos que atendían esta necesidad, lo que conllevó finalmente con el surgimiento del Plan Maestro de Desarrollo Espiritual (PDME) que está vigente hasta hoy. ${ }^{12}$

\section{Buscando una solución para el desarrollo espiritual}

En retrospectiva, ya desde 1979, George H. Akers y Robert D. Moon escribieron que "la preocupación más grande de las escuelas cristianas [adventistas] debe ser la integración del aprendizaje, la fe y la práctica en todos los aspectos del plan de estudios".13 Para que esto suceda hacía falta un proyecto que sirviera a ese propósito, y desde su perspectiva se lo atribuyeron al docente en el aula. A lo largo de los años se han publicado series de escritos de autores adventistas haciendo alusión a la necesidad de establecer medios y formas para conseguir los propósitos educacionales de las instituciones adventistas.

A inicios de los años 1990, aparecieron publicaciones en la Revista Educación Adventista en inglés y español que abordaban el tema del desarrollo de la fe y la espiritualidad de los estudiantes. Los aspectos abordados fueron principalmente el rol del docente en la integración fe y aprendizaje, ${ }^{14}$

11 George R. Knight, La visión apocalíptica y la neutralización del adventismo (Buenos Aires: Asociación Casa Editora Sudamericana, 2010), 12-13.

12 Enrique Becerra, "Hacia un plan maestro de desarrollo espiritual”, Revista Educación Adventista 9, no. 301 (1998): 3-5.

13 Robert H. Akers y Robert D. Moon, “Integrating Learning, Faith, and Practice in Christian Education: Part I", Journal of Adventist Education 42, no. 4 (1980): 17-32, http://circle.adventist.org/files/jae/en/jae198042041716.pdf (consultado: 25 de julio, 2018).

14 Bárbara Manspeaker, “¿Hice todo lo posible?”, Revista Educación Adventista 1 (1992), 9-10, http://circle.adventist.org/files/jae/sp/jae1992sp010803.pdf (consultado: 25 de julio, 2018). 
las experiencias de éxito en el aula universitaria, ${ }^{15}$ el contenido del curso, ${ }^{16}$ el papel de las guías curriculares para desarrollar la fe en los estudiantes, ${ }^{17}$ la reorientación del currículo adventista y la búsqueda de un modelo educacional propio, ${ }^{18}$ así como diversas sugerencias para hacer que la labor del docente en el aula sea más efectiva al momento de comunicar las verdades espirituales y prácticas del evangelio..$^{19}$

Posteriormente se empezó a considerar otras áreas de la institución que también deberían formar parte del proceso influenciador en pro de la vida

15 Bill Walthall, “La integración de la fe y la enseñanza en el aula universitaria, parte I: Descubrimientos de un profesor", Revista Educación Adventista 1 (1992), 18-20, http://circle.adventist.org/files/jae/sp/jae1992sp011803.pdf (consultado: 25 de julio, 2018).

16 Bill Walthall, “La integración de la fe y la enseñanza en el aula universitaria, parte II: Diseño y contenido del curso", Revista Educación Adventista 1 (1992), 21-23, http://circle.adventist.org/files/jae/sp/jae1992sp012103.pdf (consultado: 25 de julio, 2018).

17 Barry Hill, “Las guías curriculares: Medios para desarrollar la fe en alumnos de nivel secundario", Revista Educación Adventista 2 (1994), 12-19, http://circle.adventist.org/files/jae/sp/jae1994sp021205.pdf (consultado: 25 de julio, 2018).

18 George R. Knight, “¿Cuál es el conocimiento más valioso?: La educación superior adventista y su búsqueda de significado", Revista Educación Adventista 2 (1994): 4-7, http://circle.adventist.org/files/jae/sp/jae1994sp020404.pdf. (consultado: 25 de julio, 2018).

19 Véase Charleen Kurth-Wright, "Cómo hacer para que la fe forme parte de nuestro horario diario", Revista Educación Adventista 3 (1995): 25, http://circle.adventist.org/files/jae/sp/jae1995sp032503.pdf (consultado: 25 de julio, 2018); Patrik B. Morrison, "Su clase de Biblia: ¿Da vida o letargo?", Revista Educación Adventista 3 (1995): 9, http://circle.adventist.org/files/jae/sp/jae1995sp030904.pdf (consultado: 25 de julio, 2018); Raquel I. Korniejczuk y Paul S. Brandley, "Del dicho al hecho: El profesor que integra la fe y la enseñanza en la sala de clases", Revista Educación Adventista 3 (1995): 13, http://circle.adventist.org/files/jae/sp/jae1995sp031307.pdf (consultado: 25 de julio, 2018); George H. Akers, "Para fomentar la fe en la escuela cristiana", Revista Educación Adventista 3 (1995): 4, http://circle.adventist.org/files/jae/sp/jae1995sp030405.pdf (consultado: 25 de julio, 2018); Rebeca D. Becker, "¿Puede haber fe en la sala de clases de idioma y arte?", Revista Educación Adventista 3 (1995): 28, http://circle.adventist.org/files/jae/sp/jae1995sp032804.pdf (consultado: 25 de julio, 2018). 
espiritual del estudiante como la biblioteca, ${ }^{20}$ además de tener una estrategia para motivar el espíritu misionero en los estudiantes. ${ }^{21}$ Se comenzaron a desarrollar posteriormente llamados al regreso a los fundamentos de la educación adventista ${ }^{22}$ en los que se señalaba que los educadores deberían promover el "desarrollo equilibrado en cada dimensión de la vida del estudiante -intelectual, espiritual, física y social". ${ }^{23}$

Ya para 1997, se consideraba la posibilidad de hacer una evaluación de la educación superior adventista para observar cómo estaba su identidad denominacional, su sentido de responsabilidad laboral, su programa de espiritualidad y la búsqueda de la excelencia. De la espiritualidad se escribió: "Una institución cristiana debe promover y nutrir intencionalmente la vida espiritual de sus estudiantes... nada destruye más la espiritualidad como transformarla en un departamento". ${ }^{24}$

La novena edición de la revista Educación Adventista en español de 1998, dedicó todos sus artículos a tratar el desarrollo espiritual en las instituciones educativas adventistas. Al parecer los pensadores adventistas se dieron cuenta que el desarrollo espiritual no ocurría espontáneamente y que las instituciones necesitaban un plan para conseguirlo de manera deliberada. Así, concluyeron que "el crecimiento espiritual de nuestros alumnos está sobreentendido... Pero ¿enfocamos nuestros objetivos de crecimiento espiritual con la misma intencionalidad y claridad que los objetivos académi$\cos ?^{25}$

Dos años antes, el Concilio Anual de la Asociación General de los Adventistas del Séptimo Día de 1996 (el nivel más alto de su estructura organizacional) había votado un documento titulado Compromiso total con Dios. ${ }^{26}$

20 Keith Klouten, "La biblioteca de fe: Integración de la biblioteca académica en la misión del colegio", Revista Educación Adventista 4 (1995): 23, http://circle.adventist.org/files/jae/sp/jae1995sp042305.pdf (consultado: 25 de julio, 2018).

21 Dorothy Eaton Watts, “Cómo motivar a los adolescentes para participar en la Misión Global", Revista Educación Adventista 4 (1995), http://circle.adventist.org/files/jae/sp/jae1995sp042903.pdf (consultado: 25 de julio, 2018).

22 Humberto M. Rasi, “De regreso a los fundamentos adventistas", Revista Educación Adventista 5 (1996): 3-4, http://circle.adventist.org/files/jae/sp/jae1996sp050302.pdf (consultado: 25 de julio, 2018).

23 Ibíd.

24 John M. Fowler, "Evaluación de la educación adventista", Revista Educación Adventista 7 (1997): 3-4, http://circle.adventist.org/files/jae/sp/jae1997sp070302.pdf (consultado: 25 de julio, 2018).

25 Enrique Becerra, “Hacia un plan maestro”, 3-4.

26 Ibíd. 
Este documento establecía que las instituciones educativas en todos sus niveles debían "desarrollar un plan maestro de desarrollo espiritual amplio, propuesto por el personal de la institución y aprobado por su junta directiva, que identifique las verdades y valores espirituales, tanto de conocimiento como de relación, que la institución se ha comprometido a compartir con sus alumnos". ${ }^{27}$ En 1998, Dudley escribió un artículo titulado El desarrollo espiritual y el crecimiento de la fe de los alumnos de colegios y universidades adventistas en el que señaló que los métodos de presión no tenían efecto en los estudiantes, y que los valores no se transmitían sino que se elegían. ${ }^{28}$ Por tanto, era necesario desarrollar estrategias para lograr los objetivos espirituales además de un pensamiento crítico en los estudiantes, algo que ya había señalado Elena G. de White. ${ }^{29}$

En este contexto, McBride señaló que se necesitaba crear una forma de evaluar los objetivos espirituales, los mismos que debían formar parte de toda la planificación del programa educacional. ${ }^{30}$ En 1999 la Oficina de Educación de la Asociación General de los Adventistas del Séptimo Día publicó el documento A Guidebook for Creating and Implementing a Spiritual Master Plan que respondía a la necesidad de contar con una guía que permita implementar este proyecto en todas las instituciones educativas adventistas alrededor del mundo. ${ }^{31}$ En consecuencia, para las instituciones educativas en los países de Sudamérica, la División Sudamericana de los Adventistas

27 Ibíd.

28 Roger L. Dudley, “El desarrollo espiritual y el crecimiento de la fe de los alumnos de colegios y universidades adventistas", Revista Educación Adventista 9 (1998): 1014, http://circle.adventist.org/files/jae/sp/jae1998sp091005.pdf (consultado: 26 de julio, 2018).

29 "La obra de la verdadera educación consiste en desarrollar esta facultad, en educar a los jóvenes para que sean pensadores, y no meros reflectores del pensamiento de los hombres". White, La educación, 16.

30 Duane C. Mcbride, "Hacia la evaluación espiritual en los colegios y universidades adventistas", Revista Educación Adventista 9 (1998): 19-22, http://circle.adventist.org/files/jae/sp/jae1998sp091904.pdf (consultado: 26 de julio, 2018).

31 Enrique Becerra et al., A Guidebook for Creating and Implementing a Spiritual Master Plan on Seventh-day Adventist Campuses of Higher Education (Silver Spring, MD: General Conference of Seventh-day Adventists, Office of Education, 1999), https://education.adventist.org/wp-content/uploads/2017/10/Spiritual-Master-Plan-GuidebookGC.pdf (consultado: 30 de julio, 2018). 
del Séptimo Día, publicó en 2008 una versión del modelo para implementar el Plan Maestro de Desarrollo Espiritual (PMDE). ${ }^{32}$

Esta guía tenía como énfasis ayudar a que los funcionarios de las instituciones educativas participaran en la planificación eligiendo un principio, un valor y una creencia que debía promoverse a través de diversas actividades durante el año, con la finalidad que los estudiantes se lleven un claro mensaje que no se diluya con el tiempo. Actualmente las instituciones adventistas dentro de la División Sudamericana cuentan con el PMDE estandarizado, ${ }^{33}$ sin embargo, hay universidades que han desarrollado de manera particular su plan de desarrollo espiritual como la Facultad Adventista de Bahia, ${ }^{34}$ Loma Linda University Health, ${ }^{35}$ Andrews University, ${ }^{36}$ Union College. ${ }^{37}$

\section{Continúa la búsqueda por encontrar el mejor plan para el desarrollo espiritual}

Con el paso de los años existe la necesidad de establecer algún método que pueda ser medido y que permita visibilizar los resultados de los esfuerzos realizados por las instituciones adventistas respecto de su programa de educación y su impacto en la vida espiritual de sus estudiantes.

La IASD ha realizado estudios que buscaron comprender mejor el papel que desempeñan el hogar, la iglesia y la escuela en el desarrollo espiritual de los estudiantes que asistían a sus escuelas y colegios en Norteamérica.

32 Departamento de Educación de la División Sudamericana, Guía para el plan maestro de desarrollo espiritual en la educación adventista (Buenos Aires: Asociación Casa Editora Sudamericana, 2008), https://es.scribd.com/document/81725792/guia-pmde (consultado: 30 de julio, 2018).

33 Departamento de Educación de la División Sudamericana, "PMDE 2018: Generosidad - Educación Adventista", http://www.educacionadventista.com/pmde-2018-generosidad/ (consultado: 30 de julio, 2018).

34 Juan Choque et al., Plano Mestre de Desenvolvimento Espiritual: Faculdade Adventista da Bahia (Cachoeira, BA: Faculdade Adventista da Bahia, 2017).

35 Loma Linda University Health, "Loma Linda University Health Spiritual Plan 2016 2020", https://home.llu.edu/sites/home.llu.edu/files/docs/lluh-spiritual-plan-20162020.pdf (consultado: 30 de julio, 2018).

36 Andrews University, “Andrews University Spiritual Master Plan”, https://digitalcommons.andrews.edu/cgi/viewcontent.cgi ?article $=1000 \&$ context $=\mathrm{cm}$ (consultado: 30 de julio, 2018).

37 Union College, "Spiritual Master Plan Union College", https://www.ucollege.edu/spiritual-life/spiritual-master-plan (consultado: 30 de julio, 2018). 
El que se destaca fue Valuegenesis, estudio realizado entre 1990 y 1991, luego Valuegenesis II entre el 2000 y el 2001, y finalmente Valuegenesis III entre el 2010 y el 2011. El 2014, Andrea Cristina Nagy realizó un análisis de los resultados de Valuegenesis I, II y III, destacando los factores y prácticas que hacen que los estudiantes de las escuelas adventistas se desarrollen en la fe y cómo se relacionan con el hogar, la iglesia y la escuela..$^{38}$ El 2011, Glynis Madeleine Bradfield desarrolló y validó un instrumento denominado Growing Disciples Inventory (GDI) como un método para evaluar el crecimiento del discipulado en los estudiantes de nivel primario y secundario. ${ }^{39}$ Actualmente, este estudio está disponible en https://www.growingfruitfuldisciples.com y es posible que ésta sea la investigación más actual publicada sobre el desarrollo espiritual en estudiantes.

\section{Conclusión}

A lo largo de los años, la IASD ha estado buscando la mejor manera de hacer que sus programas de educación cumplan los objetivos espirituales. Quizá el mejor acierto ha sido el iniciar con una serie de investigaciones al respecto, no sólo de carácter bibliográfico sino estadístico, que ha permitido conocer la realidad del impacto que genera sobre nuestros jóvenes el programa educacional adventista. ${ }^{40}$ Sin embargo todavía queda mucho camino por recorrer. Thayer después de analizar el impacto de la educación adventista sobre los estudiantes en base a las investigaciones realizadas los últimos treinta años afirma:

Las instituciones educativas adventistas necesitan continuar y fortalecer la obra que ha producido resultados positivos relacionados con el cono-

38 Andrea Cristina Nagy, "Faith Commitments and Spiritual Influences as Correlates of Adolescents' Involvement in Service in the Valuegenesis Study" (Tesis doctoral, Andrews University, 2014), https://digitalcommons.andrews.edu/cgi/viewcontent.cgi?article $=1593 \&$ context $=$ dissertations .

39 Glynis Madeleine Bradfield, "The Development and Validation of the Growing Disciples Inventory (GDI) as a Curriculum-aligned Self-assessment for Christian Education" (Tesis doctoral, University of Stellenbosch, Sudafrica, 2011).

40 Un ejemplo de esto son algunas tesis de la Universidad Peruana Unión, tales como Juan Marcelo Zanga Céspedes, “Impacto del programa 'En los pasos de Jesús' en el desarrollo espiritual de estudiantes de la Universidad Peruana Unión, Lima, 2017" (Tesis doctoral, Universidad Peruana Unión, 2017); Israel Joselito Fernandez Mallma, "Propiedades psicométricas de la Escala de Desarrollo Espiritual (EDE) en universitarios de Juliaca y Tarapoto" (Tesis de maestría, Universidad Peruana Unión, 2030). 
cimiento de la Biblia, el logro académico, la relación con la Iglesia Adventista, y la mayoría de los resultados espirituales. No obstante, se necesita brindar mayor atención a la manera en la que las escuelas adventistas pueden ser más efectivas para que sus estudiantes cumplan objetivos relacionados con tener una relación personal con Jesús, procurar ser un adventista activo, la preocupación y acción social, y las normas adventistas de conducta. ${ }^{41}$

Por otro lado, se han encontrado escasas investigaciones relacionadas al impacto del PMDE aplicado en las instituciones adventistas sobre todo desde su creación. Es decir, existe una necesidad de evaluar el mismo PMDE como programa para medir su nivel de impacto sobre nuestros estudiantes. Instrumentos de medición de espiritualidad pueden ser útiles, así como orientar investigaciones en espiritualidad y educación. Por otro lado, la estandarización del PMDE, por ejemplo, en la División Sudamericana, es útil para unificar criterios y mantener un perfil corporativo, pero es posible que la estandarización deje brechas de necesidades específicas en las diferentes instituciones educativas adventistas debido a sus propias características. Las escasas publicaciones sobre el impacto del PMDE en los estudiantes, sobre todo en Sudamérica, muestran que el impacto de la labor adventista en este campo esta o sobre entendida o quizá necesita ser mas enfatizada por los líderes e investigadores ${ }^{42}$. En términos concretos, si la educación adventista busca "restaurar la imagen de Dios en el ser humano" deberíamos ahondar en estudios que nos permitan ver cómo impactan nuestros esfuerzos que demandan presupuesto, tiempo y recursos humanos, en nuestros estudiantes.

41 Jerome Thayer, "Treinta años de investigaciones sobre el impacto de las escuelas adventistas en los estudiantes", Revista Educación Adventista 46 (2018), https://jae.adventist.org/es/2018.4.3 (consultado el 30 de abril de 2020).

42 Se ha encontrado muy poca explicación sobre el propósito del Plan Maestro de Desarrollo Espiritual y sus objetivos específicos. Además de la guía para implementar el PMDE de la DSA de 2008, el investigador no ha encontrado mayor información sobre los objetivos actuales ni su relación entre los indicadores, los objetivos educacionales y el programa curricular.

https://doi.org/10.17162/recm.v18i1-2.1534 